\title{
On the origin of lipid asymmetry: the flip side of ion transport Guillaume Lenoir ${ }^{1}$, Patrick Williamson ${ }^{2}$ and Joost CM Holthuis ${ }^{1}$
}

\begin{abstract}
Membrane lipid asymmetry influences a multitude of cellular functions, including membrane vesiculation, cell division, and lifespan. Most cells retain the bulk of aminophospholipids to the cytosolic membrane leaflet by means of ATP-fuelled flippases or translocases. Converging lines of evidence indicate that members of the $\mathrm{P}_{4}$ subfamily of P-type ATPases catalyze aminophospholipid transport and create lipid asymmetry in late secretory and endocytic compartments. Yet P-type ATPases usually pump small cations or metal ions. Atomic structures revealed important aspects of the transport mechanism, and sequence homology indicates that this mechanism is conserved throughout the family. Consequently, understanding how $\mathrm{P}_{4}$ ATPases acquired the ability to translocate phospholipids instead of simple ions has become a major focus of interest.
\end{abstract}

\section{Addresses}

${ }^{1}$ Department of Membrane Enzymology, Bijvoet Center and Institute of Biomembranes, Utrecht University, $3584 \mathrm{CH}$ Utrecht, The Netherlands

${ }^{2}$ Department of Biology, Amherst College, Amherst, MA 010022, USA

Corresponding author: Williamson, Patrick (plwilliamson@amherst.edu) and Holthuis, Joost CM (j.c.holthuis@uu.nl)

\section{This review comes from a themed issue on Biopolymers \\ Edited by Jennifer Kohler and Jason Chin \\ Available online 5th November 2007 \\ $1367-5931 / \$$ - see front matter \\ (C) 2007 Elsevier Ltd. All rights reserved.}

Current Opinion in Chemical Biology 2007, 11:654-661

DOI 10.1016/j.cbpa.2007.09.008

\section{Introduction}

A fascinating feature of membranes in eukaryotic cells is that the different lipid species are often nonrandomly distributed across the bilayer. The paradigm for an asymmetric lipid distribution is the plasma membrane where the aminophospholipids phosphatidylserine (PS) and phosphatidylethanolamine $(\mathrm{PE})$ are mainly restricted to the cytosolic leaflet and the sphingolipids (e.g. sphingomyelin, glycosphingolipids) are enriched in the exoplasmic leaflet [1]. This asymmetry is found in some intracellular membrane systems as well and its biological significance is multifold. An asymmetric lipid arrangement provides the two sides of the bilayer with different biophysical properties that are relevant for their respective physiological roles. For instance, the tight packing of sphingolipids and sterols in the exoplasmic leaflet contributes to the barrier function of the plasma membrane and is important for membrane stability in circulating blood cells [2,3]. Conversely, the enrichment of aminophospholipids in the cytosolic leaflet of the plasma membrane and on the surface of endocytic and exocytic vesicles may help to keep these membranes in a fusion-competent state [4]. The regulated dissipation of lipid asymmetry and externalization of PS can trigger a variety of physiological responses, ranging from blood coagulation, myotube formation and sperm capacitation to phagocytic recognition, and clearance of apoptotic cells [5,6]. Furthermore, the dynamic process of lipid translocation has been implicated in membrane bending and in the biogenesis of endocytic and exocytic vesicles [7-9].

Understanding the mechanisms that create lipid asymmetry and their functional implications is an exciting and rapidly expanding field. At present, much attention is focused on a group of protein catalysts, termed flippases, which help to create lipid asymmetry by imposing selectivity and directionality on transbilayer lipid movement [10,11]. Recent work identified members of a subfamily of P-type ATPases as prime candidate flippases. The sequence organization of these so-called $\mathrm{P}_{4}$ ATPases is similar to that of the $\mathrm{Ca}^{2+}$-transporting P-type ATPase. High-resolution X-ray structures of the latter enzyme have disclosed new insights into the mechanism of Ptype ATPase-catalyzed transport. This review first highlights the current evidence for a primary role of $\mathrm{P}_{4}$ ATPases in lipid transport and asymmetry. Next, recent lessons from the $\mathrm{Ca}^{2+}$-ATPase are used to shed light on the inner workings of the $\mathrm{P}_{4}$ ATPase class of lipid pumps.

\section{Origin of lipid asymmetry}

Although phospholipids can diffuse rapidly in the lateral plane of a bilayer, they face a substantial thermodynamic barrier to 'flip' their polar headgroups through the hydrophobic membrane interior [11]. This poses a problem for biogenic membranes like the endoplasmic reticulum (ER) where phospholipid biosynthesis is mainly confined to the cytosolic leaflet. To ensure balanced growth of the membrane, half of the newly synthesized phospholipids must cross the bilayer. This led Mark Bretscher to postulate the existence of a flippase, that is, an intrinsic proteinaceous component that facilitates the energetically unfavorable migration of phospholipids across the bilayer [12]. ER flippases function independently of metabolic energy and catalyze a fast transverse movement of most phospholipid classes in both directions $\left[13,14,15^{\circ}\right]$. However, their identity is not known, and more than one protein may contribute $[16,17]$. Because ER flippases are nonspecific and nonvectorial, they produce a symmetrical lipid distribution across the bilayer. Lipid asymmetry must therefore 
be generated as membrane flows through the Golgi or upon arrival at the plasma membrane.

Lipid asymmetry cannot be explained by sidedness of lipid biosynthesis or breakdown alone and relies, at least in part, on a combination of slow flip-flop and the presence of selective lipid transporters. In contrast to the ER, nonspecific flip-flop in the plasma membrane is slow. This implies that the process of catalyzing flip-flop in the ER must be downregulated as membrane flows through the Golgi to the plasma membrane. Slow flip-flop is a prerequisite for preserving asymmetric lipid arrangements that are created by energy-dependent flippases or translocases. These activities use ATP hydrolysis to move specific lipid species against a concentration gradient. The beststudied example is the aminophospholipid translocase (APLT), which catalyzes a fast inward movement of PS and PE across the plasma membrane. Although first discovered in human erythrocytes [18], APLT activities have now been detected in the plasma membrane of many nucleated cells as well as in bovine chromaffin granules and in the trans-Golgi network (TGN) of budding yeast $\left[8,19,20^{\bullet \bullet}\right]$.

\section{The $\mathrm{P}_{\mathbf{4}}$ ATPase family of putative phospholipid translocases}

The biochemical properties defined for the APLT activity in human erythrocytes (e.g. vanadate sensitivity, high selectivity for PS) established the criteria to search for the responsible enzyme. The discovery of an APLT activity in bovine chromaffin granules led to the purification and cloning of the bovine ATPase II, now called ATP8A1 [21]. This enzyme is homologous to Drs2p, a TGN-resident protein in yeast with a role in clathrincoated vesicle formation [22,23]. ATP8A1 and Drs2p are founding members of a conserved subfamily of P-type ATPases (type IV or $\mathrm{P}_{4}$ ) that includes 5 yeast (Drs2p, Dnf1p, Dnf2p, Dnf3p, and Neo1p; Table 1) and 14 human members (ATP8A1-ATP11C) [9,24]. The relationship between $\mathrm{P}_{4}$ ATPases, APLTs, and lipid asymmetry has been the focus of considerable experimental attention.

For example, Dnf1p and Dnf2p localize to the yeast plasma membrane and their removal essentially abolishes inward translocation of NBD-labeled PS, PE, and PC analogs across the plasma membrane and causes aberrant exposure of endogenous aminophospholipids at the cell surface [25]. However, since $\Delta d n f 1 \Delta d n f 2$ mutations also perturb the endocytic pathway $[25,26]$, these data leave open the possibility that Dnf1p and Dnf2p are required to retain other proteins at the plasma membrane with a more direct role in phospholipid transport. In fact, $\mathrm{P}_{4}$ ATPases in yeast are implicated in virtually every trafficking pathway that involves ADP-ribosylation factor (ARF)dependent formation of coated vesicles [9,22,26]. Importantly, TGN membranes purified from strains that lack the Dnf proteins and contain a temperature-sensitive $d r s 2$ allele become defective in NBD-PS translocation when shifted to the nonpermissive temperature [20• $]$. These data indicate that Drs2p is directly coupled to an APLT activity and subsequent studies showed that Drs2p, together with Dnf3p, is required for maintaining aminophospholipid asymmetry in post-Golgi secretory vesicles $\left[27^{\circ}\right]$. A primary role of $\mathrm{P}_{4}$ ATPases in phospholipid translocation is further supported by the observation that the ATPase activity of ATP8A1 is selectively activated by PS and displays the same stereochemical preference for the $s n$-1,2-glycerol isomer as the APLT activity in human erythrocytes [ $\left.28^{\circ}\right]$. Moreover, a deficiency of ATP8B1, a $\mathrm{P}_{4}$ ATPase located in the canalicular membrane of hepatocytes and implicated in progressive familial intrahepatic cholestasis type 1 (FIC1) disease, is accompanied by an enhanced recovery of PS in bile [29 $9^{\circ}$.

The simplest interpretation of the available evidence is that at least some $\mathrm{P}_{4}$ ATPases actively transport phospholipids using the free energy from ATP hydrolysis. But several complications have been suggested to cloud this simple interpretation. For example, the proton ionophore CCCP has been shown to block PC transport in yeast. Although a direct effect on the transporter was not ruled out, this finding suggested that the proton electrochemical gradient across the plasma membrane in yeast might be necessary for inward phospholipid transport [30 $\left.0^{\circ}\right]$. However, the proton gradient is formed by the plasma membrane $\mathrm{H}^{+}$-ATPase Pma1p rather than Dnf1p and Dnf2p, and so how the proton gradient contributes to phospholipid transport is entirely unclear. In view of their general structural similarity to cation-transporting P-type ATPases, it has been postulated that $\mathrm{P}_{4}$ ATPases pump cations to generate a concentration gradient that subsequently drives phospholipid translocation through an

\section{Table 1}

\begin{tabular}{lllll}
\hline $\mathbf{P}_{\mathbf{4}}$ ATPase/CDC50 complexes in budding yeast & & & \\
\hline $\mathrm{P}_{4}$ ATPase & CDC50 subunit & Location & Substrates & Reference \\
\hline Dnf1p & Lem3p & PM & PS, PE, PC & {$\left[25,26,32,33,34^{\bullet \bullet}\right]$} \\
Dnf2p & Lem3p & PM & PS, PE, PC & {$\left[25,26,32,33,34^{\bullet \bullet}\right]$} \\
Dnf3p & Crf1p & TGN & PC, PE & {$\left[26,27^{\bullet}, 35\right]$} \\
Drs2p & Cdc50p & TGN & PS, PE & {$\left[20^{\bullet \bullet}, 26,27^{\bullet}, 34^{\bullet \bullet}\right]$} \\
Neo1p & Cdc50p? & Endosome & {$\left[34^{\bullet *, 47]}\right.$} \\
\hline
\end{tabular}


undefined symporter [31]. By itself, this proposal does not address the most important aspects of the model, such as which cations are transported, and how the model might give rise to lipid specificity. Nevertheless, one aspect of this model deserves mention: genetic disruption of the putative symporter should also block phospholipid transport and phenocopy $d n f$ and $d r s 2$ mutations. Interestingly, mutations in members of the CDC50 protein family produce just such phenotypes $\left[32,33,34^{\bullet \bullet}\right]$. This family includes three yeast (Cdc50p, Lem3p/Ros3p, and Crf1p) and three human proteins (CDC50A, CDC50B, and CDC50C) that consist of two transmembrane segments and an exoplasmic, potentially glycosylated loop. These proteins are not independent entities in the membrane, but rather form heteromeric complexes with $\mathrm{P}_{4}$ ATPases (Table 1). The correct assembly of these complexes is required for export from the ER $\left[34^{\bullet \bullet}, 35\right]$, which led to the suggestion that CDC50 proteins function as chaperones involved in the proper localization of $\mathrm{P}_{4}$ ATPases. An alternative possibility is that CDC50 proteins are intrinsic components of the $\mathrm{P}_{4}$ ATPase transport machinery.

\section{Mechanism of P4 ATPase-catalyzed phospholipid transport}

Based on sequence homology, the P-type ATPase superfamily can be divided into five major branches, named $\mathrm{P}_{1}-\mathrm{P}_{5}$ [36]. In addition to $\mathrm{P}_{4}$ ATPases, these include softtransitional-metal-translocating ATPases $\left(\mathrm{P}_{1 \mathrm{~B}}\right), \mathrm{Ca}^{2+}$. ATPases $\left(\mathrm{P}_{2 \mathrm{~A} / \mathrm{B}}\right), \mathrm{Na}^{+} / \mathrm{K}^{+}$-ATPases and $\mathrm{H}^{+} / \mathrm{K}^{+}$-ATPases
$\left(\mathrm{P}_{2 \mathrm{C}}\right), \mathrm{H}^{+}$-ATPases $\left(\mathrm{P}_{3 \mathrm{~B}}\right)$, and a class of ATPases whose substrate specificity is not known $\left(\mathrm{P}_{5}\right)$. P-type ATPases are $70-150 \mathrm{kDa}$ polytopic membrane proteins that usually contain 10 membrane-spanning helices (M1-M10; Figure 1a). They share a common enzymatic mechanism in which ATP hydrolysis is used to transport ligands, usually cations, across a membrane. The name 'P-type' comes from the acid-stable, phosphorylated Asp residue that forms during the enzyme's transport cycle. At least two conformations exist, E1 and E2, with conformational changes being accompanied by translocation of the ligand.

\section{P-type ATPase transport cycle}

The transport cycle of $\mathrm{Ca}^{2+}$-ATPases and $\mathrm{Na}^{+} / \mathrm{K}^{+}$ATPases has been worked out in detail $[31,37,38]$. It starts with binding of a ligand (two $\mathrm{Ca}^{2+}$ ions in the case of $\mathrm{P}_{2 \mathrm{~A}}$ ATPases) to a conformation called E1, which has a high-affinity site in the membrane domain directly accessible from the inside (cytosolic side; Figure 1b). Binding of the ligand allows the crucial Asp residue to become phosphorylated by $\mathrm{Mg}^{2+}$-ATP. The resulting intermediate, called E1P, is of high energy, because it can react readily with ADP to reform an ATP molecule. Formation of the E1P intermediate results in 'occlusion' of the ligand (i.e. blocking ligand access to the cytosol). With the release of ADP follows the downhill transition of the E1P intermediate to form the low-energy phosphorylated E2P intermediate and discharge of the ligand to the

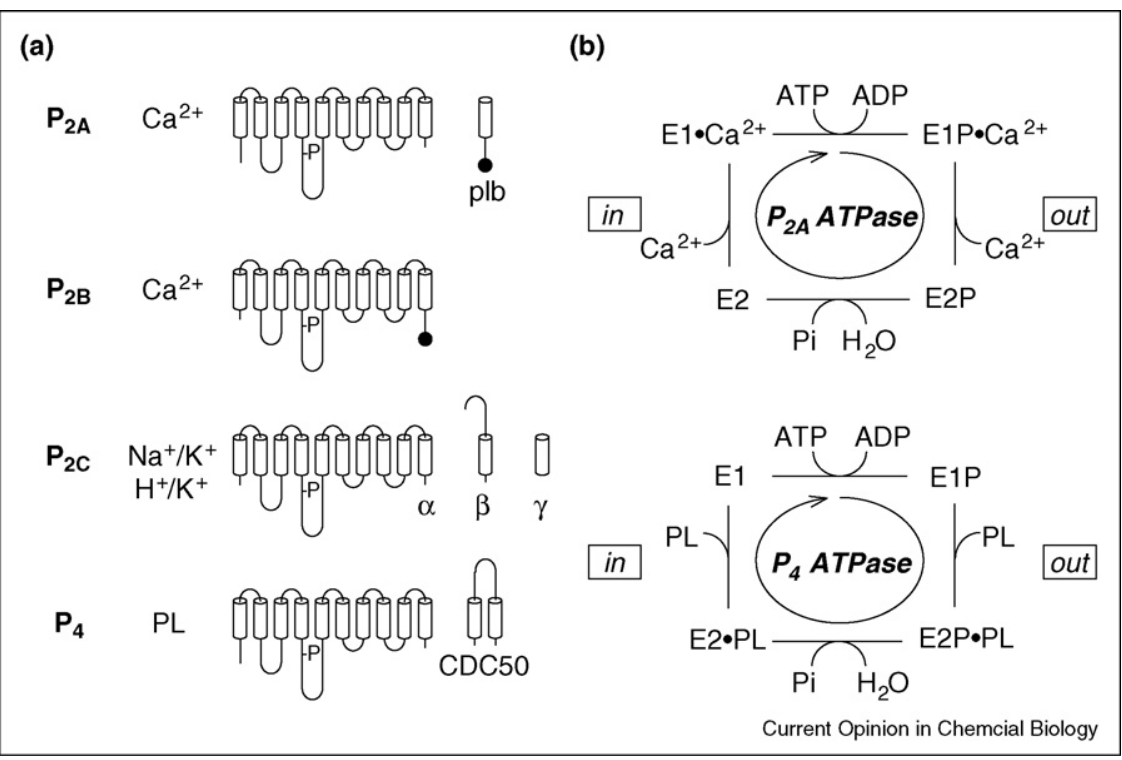

Membrane topology and transport cycle of $P_{2}$ and $P_{4}$ ATPases. (a) Substrate specificity, topology, and accessory subunits of $P_{2}$ and $P_{4}$ ATPases. Transmembrane helices (TM) are shown as white cylinders. The phosphorylation site is indicated in the large, central cytoplasmic loop, connecting TM4 and TM5. Regulatory phosphorylation sites are displayed as black dots. Plb: phospholamban; PL: phospholipids. (b) Simplified scheme of the transport cycle of $\mathrm{P}_{2 \mathrm{~A}}$ and $\mathrm{P}_{4}$ ATPases, according to the E1/E2 model. Note that $\mathrm{P}_{2 \mathrm{~A}}$ ATPases translocate two Ca ${ }^{2+}$ ions per hydrolyzed ATP molecule, and pump two to three $\mathrm{H}^{+}$ions, which bind to the E2P intermediate, in the opposite direction (not shown). Whether $\mathrm{P}_{4}$ ATPase-catalyzed PL transport is coupled to counter-transport of another ligand is not known. 
outside (exoplasmic/luminal side). The ligand-binding site now has high affinity for a counter-transported ligand (two to three $\mathrm{H}^{+}$ions for $\mathrm{P}_{2 \mathrm{~A}}$ ATPases), which binds from the outside. Hydrolysis of the phosphorylated Asp results in the E2 state. $\mathrm{Mg}^{2+}$ and inorganic phosphate $(\mathrm{Pi})$ dissociate and the enzyme reverts to the $\mathrm{E} 1$ state, in which the counter-transported ligand is released into the cytosol. As discussed below, $\mathrm{Ca}^{2+}$-ATPases and $\mathrm{P}_{4}$ ATPases display a remarkable degree of structural similarity. This implies that they share the same basic mechanism of ATP-fuelled ligand transport. $\mathrm{P}_{4}$ ATPases catalyze the translocation of phospholipids from the exoplasmic to the cytosolic leaflet. This would predict that the phospholipid ligand in $\mathrm{P}_{4}$ ATPases binds to the phosphoenzyme intermediate E2P as opposed to the $\mathrm{Ca}^{2+}$ ion in $\mathrm{P}_{2 \mathrm{~A}}$ ATPases, which binds to $\mathrm{E} 1$ (Figure 1b). This concept is consistent with the finding that, while $\mathrm{Ca}^{2+}$ triggers a rapid phosphorylation of $\mathrm{P}_{2 \mathrm{~A}}$ ATPases, PS induces a fast dephosphorylation of the phosphoenzyme intermediate of the $\mathrm{P}_{4}$ ATPase, ATP8A1 [39].

\section{Lessons from a calcium pump}

A recent series of high-resolution X-ray structures allowed a $3 \mathrm{D}$ reconstruction of the transport cycle of the rabbit muscle $\mathrm{P}_{2 \mathrm{~A}} \mathrm{Ca}^{2+}$-ATPase, SERCA1 [31,40, $41^{\circ}$ ] (Figure 2). $\mathrm{Ca}^{2+}$ binding and dissociation are accompanied by piston-like movements and rotations of transmembrane helices M4-M6, which, together with M8, contain the $\mathrm{Ca}^{2+}$-coordinating side chains. These rearrangements are mechanically linked with reciprocating movements of the cytosolic $\mathrm{P}$ (phosphorylation), $\mathrm{N}$ (nucleotide binding), and A (actuator) domains, which, in turn, affect the chemical reactions in the catalytic center. The A domain is directly connected to M1-M3 and the $\mathrm{P}$ domain to the M4 and M5 helices. The $\mathrm{N}$ domain, a long insertion between two parts forming the $\mathrm{P}$ domain, contains the adenosine-binding site, whereas the $\gamma$-phosphate reacts with the Asp residue in the $\mathrm{P}$ domain. The A domain functions as the 'actuator' of the gating mechanism that regulates $\mathrm{Ca}^{2+}$ binding and release. Although mostly segregated from the other cytosolic domains in E1, the A domain rotates in the rate-limiting

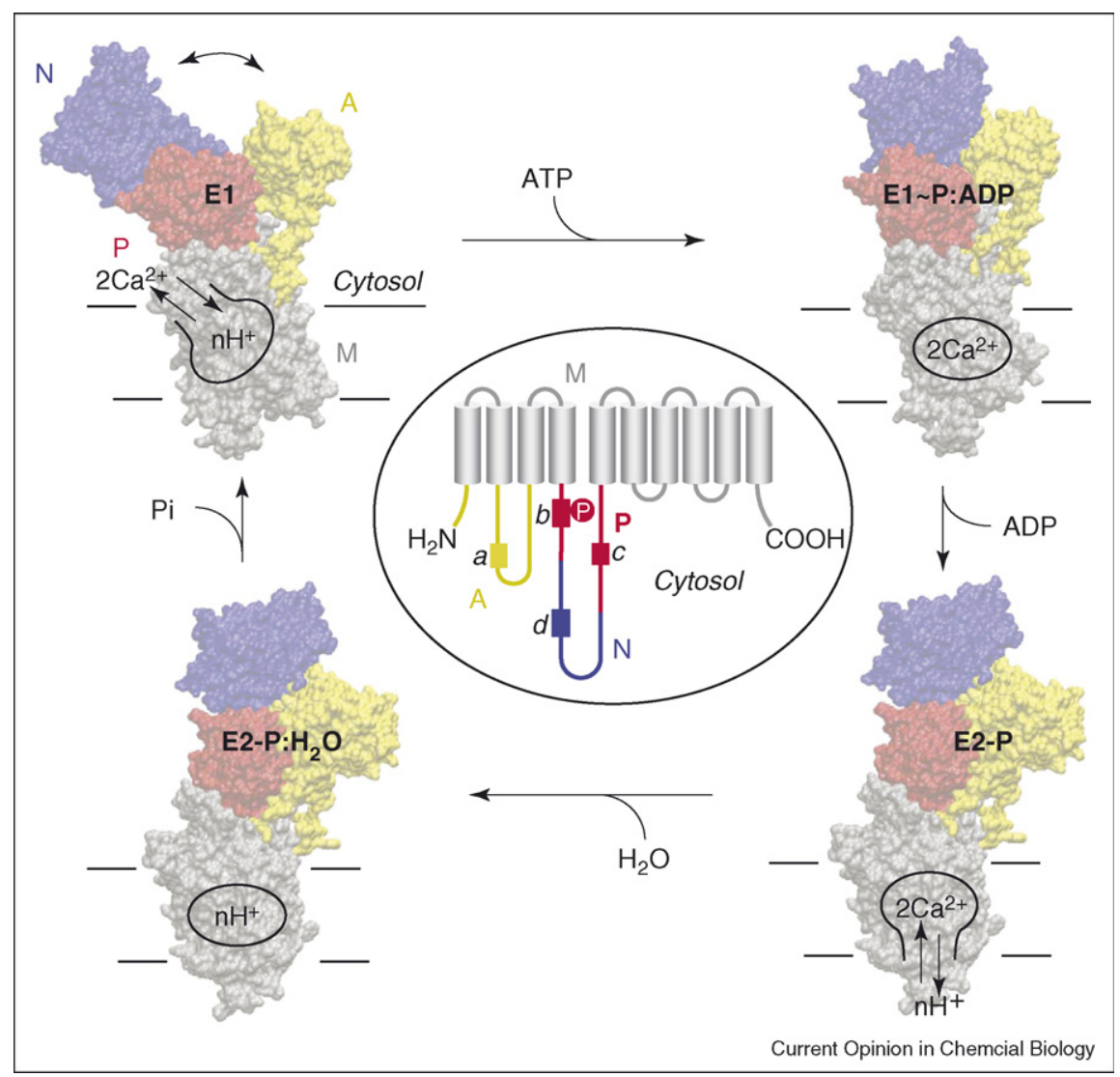

$3 \mathrm{D}$ reconstruction of the reaction cycle of the $\mathrm{P}_{2 \mathrm{~A}} \mathrm{Ca}^{2+}$-ATPase, SERCA1. Key functional intermediates and their structural correlates are shown. Blue, $N$ domain; red, $P$ domain; yellow, A domain; gray, $\mathrm{M}$ domain. Conserved sequence motifs $(a-d)$ present in cytosolic $A, P$, and $N$ domains of $\mathrm{P}_{2}$ and $\mathrm{P}_{4}$ ATPases are marked in the topological model and correspond to those displayed in Table 2. Protein Data Bank (PDB) accession codes: 1SU4 (E1); 1T5T (E1 P:ADP); 1XP5 (E2-P: $\left.\mathrm{H}_{2} \mathrm{O}\right)$. Note that the structure of E2-P is unknown, but believed to closely resemble that of E2-P: $\mathrm{H}_{2} \mathrm{O}$. This figure has been modified from [42]. 
Table 2

Conserved sequence motifs in $\mathrm{P}_{2}$ and $\mathrm{P}_{4}$ ATPases

\begin{tabular}{|c|c|c|c|c|c|}
\hline P-type & ATPase & motif $a^{1}$ & motif $\boldsymbol{b}$ & motif $c$ & motif $\boldsymbol{d}$ \\
\hline \multirow{2}{*}{$P_{2 A}$} & SERCA1a $\left(h s^{2}\right)$ & TGES & VICSDKTGLT & TGDNK & 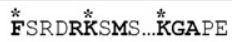 \\
\hline & $\operatorname{Pmr} 1 \mathrm{p}\left(s c^{2}\right)$ & TGES & VICSDKTGLT & TGDSE & FNSKRKLMA...KGAFE \\
\hline \multirow{4}{*}{$\mathrm{P}_{4}$} & ATP8A1 $(h s)$ & DGET & $\underline{Y} I \underline{F} S D K T G L T$ & TGDKQ & FTSARKRMS...KGADT \\
\hline & ATP8B1 (hs) & DGET & $\bar{Y} I \bar{F}$ SDKTGLT & TGDKK & FNSDRKRMS...KGADT \\
\hline & Drs2p (sc) & DGET & $\overline{\mathrm{Y}} I \overline{\mathrm{F}} \mathrm{SDKTGLT}$ & TGDRQ & FNSTRKRMS...KGA $\overline{D T}$ \\
\hline & Dnf2p (sc) & DGET & $\underline{\bar{Y}} I \overline{\bar{F}} \mathrm{SDKTGLT}$ & TGDKV & FNSSRKRMS...KGA $\overline{D T}$ \\
\hline
\end{tabular}

Motifs $(a-d)$ correspond to those displayed in topological model of Figure 2. Motif $b$ contains the invariant phosphorylated aspartic acid (gray background). Residues critical for ATP binding (motif $d$ ) are marked by an asterisk. Abbreviations: hs, Homo sapiens; sc, Saccharomyces cerevisiae.

E1P to E2P transition to bring a highly conserved TGES loop into the vacated ATP-binding site. This allows the glutamate in TGES to execute its crucial role in E2P dephosphorylation, which presumably consists of coordinating the attacking water molecule $\left[42,43^{\circ}\right]$.

Although no structural information is available for $\mathrm{P}_{4}$ ATPases, some insight into their working mechanism can be gained indirectly by taking advantage of the similarities between $\mathrm{P}_{2 \mathrm{~A}}$ and $\mathrm{P}_{4}$ ATPases. In addition to a common transmembrane domain organization, $\mathrm{P}_{2 \mathrm{~A}}$ and $\mathrm{P}_{4}$ ATPases display a clear overall sequence homology. About 230 of the roughly 1000 residues are invariant, with identical side chains or conservative substitutions in equivalent positions. Most invariant residues are found in the cytosolic domains. Shared sequence motifs include the canonical phosphorylation site in the $\mathrm{P}$ domain, the nucleotide-binding site in the $\mathrm{N}$ domain, and a TGES-related sequence in the A domain (Table 2).
Consequently, the reactions of ATP binding, phosphoryl transfer and hydrolysis, and the mechanical transduction of the energy released in this process to the ligandbinding site are probably very similar between $\mathrm{P}_{2 \mathrm{~A}}$ and $\mathrm{P}_{4}$ ATPases. The membrane (M) domain of $\mathrm{P}_{2 \mathrm{~A}}$ ATPases contains two $\mathrm{Ca}^{2+}$-binding sites that consist of a congregation of glutamates and aspartates whose anionic carboxyl groups serve to neutralize the charge of the $\mathrm{Ca}^{2+}$ ion (Figure 3, right panel). In $\mathrm{P}_{4}$ ATPases, these anionic residues have been replaced by a mixture of hydrophobic and polar uncharged residues [21,44]. Although this change in composition may hint at the transport of amphipathic molecules, it is difficult to envision how the binding site at the center of a 10-helix bundle can adapt to translocate both metal ions and phospholipids. Flippases must provide a sizeable hydrophilic pathway for the bulky lipid headgroup to pass through the membrane as well as accommodate the hydrophobic nature of the lipid backbone.

\section{Figure 3}

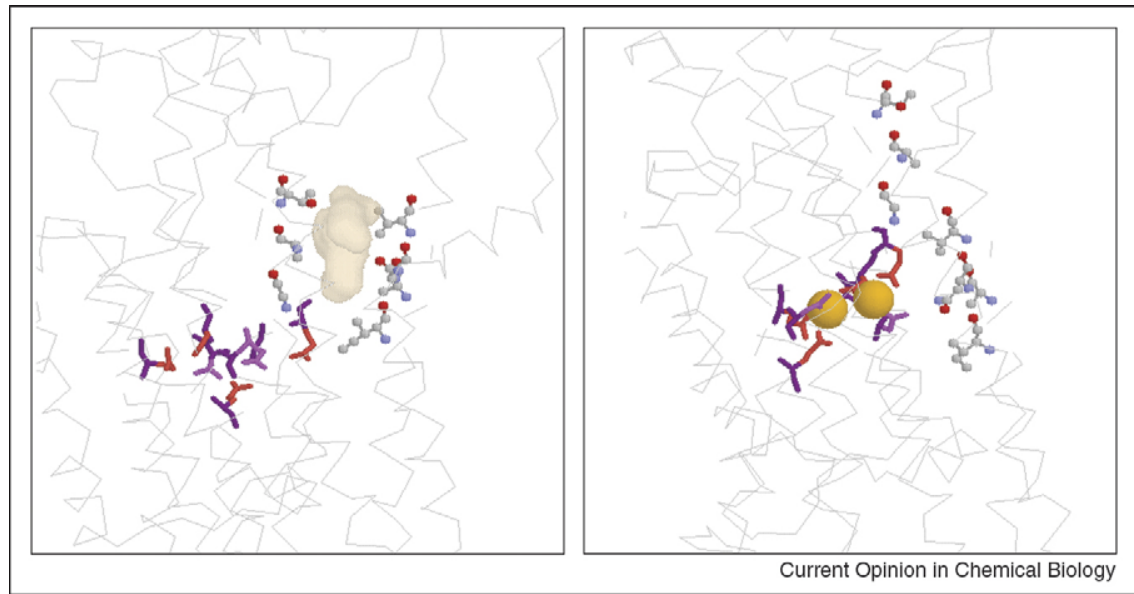

Cation and phospholipid-binding residues in the chicken muscle $\mathrm{Ca}^{2+}$-ATPase, SERCA1. Amino acids contacting the bound phospholipid in the E2 conformation (197, A100, N101, V104, G310, A313, and T316) are shown in ball and stick representation; amino acids contacting the two bound $\mathrm{Ca}^{2+}$ ions (E309, N768, E771, N796, T799, and D800) are shown in stick representation. Cytosolic side is at the top. Left: E2 conformation (2AGV), with the bound phospholipid shown in surface representation. Right: E1 conformation (1SU4), with bound $\mathrm{Ca}^{2+}$ shown as spheres. Images created with Protein Explorer (www.proteinexplorer.org). 


\section{A primordial lipid-binding site}

The high-resolution structure for a BHQ-stabilized E2 conformer of the $\mathrm{Ca}^{2+}$-ATPase SERCA [ $45^{\circ}$ ] revealed the presence of a relatively tightly bound, nonexchangeable phospholipid between the M2 and M4 helices (Figure 3, left panel). There are several thought-provoking aspects to this bound phospholipid. One is that the headgroup of the phospholipid, modeled as the aminophospholipid PE, is oriented toward the cytosolic surface, with the headgroup in a cavity. There are no interactions between the headgroup and the protein; rather, the sidechain interactions are primarily at the level of the glycerol backbone of the phospholipid, reminiscent of the stereospecificity of $\mathrm{P}_{4}$ ATPases for the same region [28]. Moreover, the phospholipid is not present in the E1 structure: the cavity that housed the headgroup is gone, and the residues that interacted with the backbone are dispersed by the same movements of the transmembrane helices that create the binding sites for $\mathrm{Ca}^{2+}$ (Figure 3, right panel). Obara et al. note that the PE probably moves into this position in the $\mathrm{E} 2 \mathrm{P} / \mathrm{E} 2$ transition, and then is ejected (on the cytosolic side) in the E2/E1 transition $\left[45^{\circ}\right]$. These events are strikingly reminiscent of those predicted for the phospholipid transport cycle of $\mathrm{P}_{4}$ ATPases. To produce vectorial transport would require only that refilling the binding site at the E2P/E2 transition be accomplished using a phospholipid from the luminal surface. Of course, this step is the one that requires movement of the headgroup through the membrane interior, and there is no obvious pathway in the $\mathrm{Ca}^{2+}$-ATPase structure for such movement (nor would one be expected - the $\mathrm{Ca}^{2+}$ ATPase does not transport phospholipids). To bring the $\mathrm{PE}$ to this position from a bound state at the luminal surface in the E2P conformation of the $\mathrm{P}_{4}$ ATPases would probably require additional molecular machinery.

\section{Role of $\mathbf{P}_{\mathbf{4}}$ ATPase subunits}

$\mathrm{P}_{4}$ ATPases are capable of discriminating phospholipids based on atoms in both the headgroup and lipid backbone $\left[25,28^{\circ}\right]$. Moreover, $\mathrm{P}_{4}$ ATPases in yeast display striking differences in transport specificity. Although Drs2p primarily serves as a PS transporter [20*0], Dnf3p preferentially recognizes PG $\left[27^{\circ}\right]$ (Table 1 ). Dnf1p and Dnf2p, on the other hand, display no such preferences and seem to transport PS, PE, and PG with similar efficiencies [25]. Interestingly, Dnf1p and Dnf2p form heteromeric complexes with a common CDC50-binding partner, that is, Lem3p [34*•]. By contrast, Drs2p and Dnf3p interact with different CDC50 proteins, namely Cdc50p and Crf1p, respectively $\left[34^{\bullet \bullet}, 35\right]$ (Table 1 ). This arrangement unlikely reflects a primary role of CDC50 proteins in $\mathrm{P}_{4}$ ATPase trafficking since both Drs2p and Dnf3p are TGN-associated proteins and cycle through the plasma membrane by entering a common class of secretory vesicles $\left[25,26,27^{\circ}\right]$. A more attractive option is that CDC50 proteins help to specify $\mathrm{P}_{4}$ ATPase-catalyzed lipid transport.
CDC50 proteins contribute two additional helices to the 10-helix bundle, which forms the $\mathrm{M}$ domain of the transporters. This augmentation of the structure might actively contribute to the transport specificity of $\mathrm{P}_{4}$ ATPases in several ways. For instance, creation of a high-affinity phospholipid-binding site may require CDC50-induced conformational changes in the 10-helix bundle of the $\mathrm{P}_{4}$ ATPase, analogous to the role of the accessory subunits in $\mathrm{Na}^{+} / \mathrm{K}^{+}$-ATPases and $\mathrm{H}^{+} / \mathrm{K}^{+}$-ATPases, which modulate the affinity of these transporters for $\mathrm{Na}^{+}$and $\mathrm{K}^{+}[38,46]$. Alternatively, CDC50 proteins may contribute more directly to the formation of a specific phospholipid-binding site by completing the binding site for this large substrate. Indeed, an active role of CDC50 proteins in $\mathrm{P}_{4}$ ATPasecatalyzed phospholipid transport may explain our recent observation that $\mathrm{P}_{4}$ ATPase/CDC50 interactions are sensitive to mutation of the catalytically important Asp residue [our unpublished results]. This finding suggests that the transporter/subunit affinity fluctuates during the transport cycle and that CDC50 proteins interact most strongly with a phosphorylated intermediate of the ATPase, which is the conformation that becomes loaded with the phospholipid ligand.

\section{Conclusions}

Genetic and biochemical evidence indicates that $\mathrm{P}_{4}$ ATPases are the best candidate flippases identified till date and serve a primary role in the generation of aminophospholipid asymmetry. Yet $\mathrm{P}_{4}$ ATPases belong to the superfamily of P-type pumps whose members usually translocate small cations or metal ions. Atomic structures and homology models revealed that fundamental aspects of the transport mechanism are conserved throughout the family. Therefore, a challenging problem is to understand how an ion pump evolved into a flippase. Here we postulated two basic concepts that may serve as a useful guide. One is the idea that $\mathrm{Ca}^{2+}$-ATPases may contain a primordial phospholipid-binding site in the periphery of the 10-helix bundle in addition to the more centrally located cation-binding sites. The putative phospholipidbinding site is transient, with the phospholipid moving in during the E2P/E2 transition and moving out in the E2/ $\mathrm{E} 1.2 \mathrm{Ca}^{2+}$ step of the reaction cycle. Intriguingly, this sequence of events matches those predicted for $\mathrm{P}_{4}$ ATPases, which should become loaded with phospholipid ligand in E2P. The other concept is that, due to evolutionary constraints, the 10-helix bundle of a $\mathrm{P}_{4}$ ATPase may not be sufficient to provide for the entire pathway by which phospholipids translocate through the membrane interior. To drive vectorial lipid transport, $\mathrm{P}_{4}$ ATPases might need additional molecular machinery. The CDC50 proteins are obvious candidates for such additional machinery. It appears that CDC50-P ATPases interactions are dependent on the conformation of the transporter and coincide with loading of the phospholipid substrate. By contributing two additional helices to the 10-helix bundle, CDC50 proteins may help to 
complete a pathway for lipid translocation as well as contribute directly to the transport specificity of $\mathrm{P}_{4}$ ATPases. Obviously, this arrangement would provide an attractive target for regulation.

\section{Acknowledgements}

We wish to thank Catheleyne Puts for valuable comments on the manuscript. This work was supported by grants from the Dutch Organization of Sciences (NWO-CW), the Utrecht High Potential Program (to JH), and the National Science Foundation (to PW).

\section{References and recommended reading}

Papers of particular interest, published within the annual period of review, have been highlighted as:

- of special interest

•• of outstanding interest

1. Zachowski A: Phospholipids in animal eukaryotic membranes: transverse asymmetry and movement. Biochem J 1993, 294(Pt 1):1-14.

2. Antia R, Schlegel RA, Williamson $P$ : Binding of perforin to membranes is sensitive to lipid spacing and not headgroup. Immunol Lett 1992, 32:153-157.

3. Holthuis JC, Pomorski T, Raggers RJ, Sprong H, van Meer G: The organizing potential of sphingolipids in intracellular membrane transport. Physiol Rev 2001, 81:1689-1723.

4. Kinnunen PK, Holopainen JM: Mechanisms of initiation of membrane fusion: role of lipids. Biosci Rep 2000, 20:465-482.

5. Schlegel RA, Williamson P: Phosphatidylserine, a death knell. Cell Death Differ 2001, 8:551-563.

6. Balasubramanian K, Schroit AJ: Aminophospholipid asymmetry: a matter of life and death. Annu Rev Physiol 2003, 65:701-734.

7. Devaux PF: Is lipid translocation involved during endo- and exocytosis? Biochimie 2000, 82:497-509.

8. Pomorski T, Holthuis JC, Herrmann A, van Meer G: Tracking down lipid flippases and their biological functions. J Cell Sci 2004, 117:805-813.

9. Graham TR: Flippases and vesicle-mediated protein transport. Trends Cell Biol 2004, 14:670-677.

10. Daleke DL: Phospholipid flippases. J Biol Chem 2007, 282:821-825.

11. Holthuis JC, Levine TP: Lipid traffic: floppy drives and a superhighway. Nat Rev Mol Cell Biol 2005, 6:209-220.

12. Bretscher MS: Some aspects of membrane structure. In Perspectives in Membrane Biology. Edited by Estrada OS, Gitler C. Academic Press; 1974:3-24.

13. Buton $X$, Morrot $G$, Fellmann $P$, Seigneuret M: Ultrafast glycerophospholipid-selective transbilayer motion mediated by a protein in the endoplasmic reticulum membrane. $J$ Biol Chem 1996, 271:6651-6657.

14. Vehring S, Pakkiri L, Schroer A, Alder-Baerens N, Herrmann A, Menon AK, Pomorski T: Flip-flop of fluorescently labeled phospholipids in proteoliposomes reconstituted with yeast microsomal proteins. Eukaryot Cell 2007, 6:1625-1634.

15. Papadopulos A, Vehring S, Lopez-Montero I, Kutschenko L,

- $\quad$ Stockl M, Devaux PF, Kozlov M, Pomorski T, Herrmann A: Flippase activity detected with unlabeled lipids by shape changes of giant unilamellar vesicles. J Biol Chem 2007, 282:15559-15568.

This study is the first demonstration that the dynamics of GUV shape changes can be used to monitor protein-mediated translocation of natural lipid substrates.

16. Chang QL, Gummadi SN, Menon AK: Chemical modification identifies two populations of glycerophospholipid flippase in rat liver ER. Biochemistry 2004, 43:10710-10718.
17. Kol MA, de Kroon Al, Killian JA, de Kruijff B: Transbilayer movement of phospholipids in biogenic membranes. Biochemistry 2004, 43:2673-2681.

18. Seigneuret M, Devaux PF: ATP-dependent asymmetric distribution of spin-labeled phospholipids in the erythrocyte membrane: relation to shape changes. Proc Natl Acad Sci US A 1984, 81:3751-3755.

19. Zachowski A, Henry JP, Devaux PF: Control of transmembrane lipid asymmetry in chromaffin granules by an ATP-dependent protein. Nature 1989, 340:75-76.

20. Natarajan P, Wang J, Hua Z, Graham TR: Drs2p-coupled

-• aminophospholipid translocase activity in yeast Golgi membranes and relationship to in vivo function. Proc Natl Acad Sci U S A 2004, 101:10614-10619.

This study provides some of the strongest evidence till date that $\mathrm{P}_{4}$ ATPases function as lipid translocases.

21. Tang X, Halleck MS, Schlegel RA, Williamson P: A subfamily of P-type ATPases with aminophospholipid transporting activity. Science 1996, 272:1495-1497.

22. Chen CY, Ingram MF, Rosal PH, Graham TR: Role for Drs2p, a P-type ATPase and potential aminophospholipid translocase, in yeast late Golgi function. J Cell Biol 1999 , 147:1223-1236.

23. Gall WE, Geething NC, Hua Z, Ingram MF, Liu K, Chen SI, Graham TR: Drs2p-dependent formation of exocytic clathrincoated vesicles in vivo. Curr Biol 2002, 12:1623-1627.

24. Paulusma CC, Oude Elferink RP: The type 4 subfamily of P-type ATPases, putative aminophospholipid translocases with a role in human disease. Biochim Biophys Acta 2005, 1741:11-24.

25. Pomorski T, Lombardi R, Riezman H, Devaux PF, van Meer G, Holthuis JC: Drs2p-related P-type ATPases Dnf1p and Dnf2p are required for phospholipid translocation across the yeast plasma membrane and serve a role in endocytosis. Mol Biol Cell 2003, 14:1240-1254.

26. Hua Z, Fatheddin P, Graham TR: An essential subfamily of Drs2p-related P-type ATPases is required for protein trafficking between Golgi complex and endosomal/vacuolar system. Mol Biol Cell 2002, 13:3162-3177.

27. Alder-Baerens N, Lisman Q, Luong L, Pomorski T, Holthuis JC:

- $\quad$ Loss of P4 ATPases Drs2p and Dnf3p disrupts aminophospholipid transport and asymmetry in yeast postGolgi secretory vesicles. Mol Biol Cell 2006, 17:1632-1642.

This study provides evidence for striking differences in lipid specificity among $\mathrm{P}_{4}$ ATPase family members.

28. Paterson JK, Renkema K, Burden L, Halleck MS, Schlegel RA,

- Williamson P, Daleke DL: Lipid specific activation of the murine P4-ATPase Atp8a1 (ATPase II). Biochemistry 2006 , 45:5367-5376.

This study reports that translocase activity has rather odd chemical and stereochemical specificities. The ATPase activity of purified, recombinan ATPase8A1 is stimulated by phospholipids with those same specificities.

29. Paulusma CC, Groen A, Kunne C, Ho-Mok KS, Spijkerboer AL,

- Rudi de Waart D, Hoek FJ, Vreeling H, Hoeben KA, van Marle J et al:: Atp8b1 deficiency in mice reduces resistance of the canalicular membrane to hydrophobic bile salts and impairs bile salt transport. Hepatology 2006, 44:195-204.

This paper provides evidence that Atp8b1 deficiency dissipates lipid asymmetry in the canalicular membrane, which results in enhanced cholesterol extraction by bile salts and a reduced activity of the bile salt transporter.

30. Stevens HC, Nichols JW: The proton electrochemical gradient

- $\quad$ across the plasma membrane of yeast is necessary for phospholipid flip. J Biol Chem 2007, 282:17563-17567.

This paper reports that there are few inhibitors of lipid translocation. The proton ionophore CCCP seems to be one of them.

31. Kuhlbrandt W: Biology, structure and mechanism of P-type ATPases. Nat Rev Mol Cell Biol 2004, 5:282-295.

32. Kato U, Emoto K, Fredriksson C, Nakamura H, Ohta A, Kobayashi T, Murakami-Murofushi K, Umeda M: A novel membrane protein, Ros3p, is required for phospholipid translocation across the plasma membrane 
in Saccharomyces cerevisiae. J Biol Chem 2002, 277:37855-37862

33. Hanson PK, Malone L, Birchmore JL, Nichols JW: Lem3p is essential for the uptake and potency of alkylphosphocholine drugs, edelfosine and miltefosine. J Biol Chem 2003, 278:36041-36050.

34. Saito K, Fujimura-Kamada K, Furuta N, Kato U, Umeda M,

-• Tanaka K: Cdc50p, a protein required for polarized growth, associates with the Drs2p P-type ATPase implicated in phospholipid translocation in Saccharomyces cerevisiae. Mol Biol Cell 2004, 15:3418-3432.

This paper shows that CDC50/LEM3 protein family members form heteromeric complexes with and are required for ER export of $\mathrm{P}_{4}$ ATPases.

35. Furuta N, Fujimura-Kamada K, Saito K, Yamamoto T, Tanaka K: Endocytic recycling in yeast is regulated by putative phospholipid translocases and the Ypt31p/32p-Rcy1p pathway. Mol Biol Cell 2007, 18:295-312.

36. Axelsen KB, Palmgren MG: Evolution of substrate specificities in the P-type ATPase superfamily. $\mathrm{J} \mathrm{Mol} \mathrm{Evol}$ 1998, 46:84-101.

37. Stokes DL, Green NM: Structure and function of the calcium pump. Annu Rev Biophys Biomol Struct 2003, 32:445-468.

38. Jorgensen PL, Hakansson KO, Karlish SJ: Structure and mechanism of Na,K-ATPase: functional sites and their interactions. Annu Rev Physiol 2003, 65:817-849.

39. Ding J, Wu Z, Crider BP, Ma Y, Li X, Slaughter C, Gong L, Xie XS: Identification and functional expression of four isoforms of ATPase II, the putative aminophospholipid translocase. Effect of isoform variation on the ATPase activity and phospholipid specificity. J Biol Chem 2000, 275:23378-23386.

40. Toyoshima C, Inesi G: Structural basis of ion pumping by $\mathbf{C a}^{2+}$ ATPase of the sarcoplasmic reticulum. Annu Rev Biochem 2004, 73:269-292.
41. Moller JV, Nissen P, Sorensen TL, le Maire M: Transport mechanism of the sarcoplasmic reticulum $\mathrm{Ca}^{2+}$-ATPase pump. Curr Opin Struct Biol 2005, 15:387-393.

This study is an excellent review providing a comprehensive 3D reconstruction of the transport cycle of the $\mathrm{Ca}^{2+}$-ATPase.

42. Clausen JD, Vilsen B, Mclntosh DB, Einholm AP, Andersen JP: Glutamate-183 in the conserved TGES motif of domain A of sarcoplasmic reticulum $\mathrm{Ca}^{2+}$-ATPase assists in catalysis of E2/E2P partial reactions. Proc Natl Acad Sci U S A 2004, 101:2776-2781.

43. Anthonisen AN, Clausen JD, Andersen JP: Mutational analysis of - the conserved TGES loop of sarcoplasmic reticulum $\mathrm{Ca}^{2+}$ ATPase. J Biol Chem 2006, 281:31572-31582.

This study reports that site-specific mutagenesis and careful measurements of part reactions provide clear experimental support for a model of how the TGES consensus sequence participates in the ATPase reaction cycle.

44. Schlegel RA, Halleck MS, Williamson P: Phospholipid transporters in the brain. In Nutrition and Biochemistry of Phospholipids. Edited by Szuhaj BF, van Nieuwenhuyzen W. Champaign, IL: American Oil Chemists Society Press; 2003:1-13.

45. Obara K, Miyashita N, Xu C, Toyoshima I, Sugita Y, Inesi G,

- Toyoshima C: Structural role of countertransport revealed in $\mathbf{C a}(2+)$ pump crystal structure in the absence of $\mathbf{C a}(2+)$. Proc Natl Acad Sci U S A 2005, 102:14489-14496.

This study reports that the higher resolution of $B H Q$-stabilized E2 form of the $\mathrm{Ca}^{2+}$-ATPase reveals, among other things, several phospholipid molecules, one of which appears to be specific to this conformation.

46. Geering K: Function of FXYD proteins, regulators of $\mathbf{N a}$, K-ATPase. J Bioenerg Biomembr 2005, 37:387-392.

47. Wicky S, Schwarz H, Singer-Kruger B: Molecular interactions of yeast Neo1p, an essential member of the Drs2 family of aminophospholipid translocases, and its role in membrane trafficking within the endomembrane system. Mol Cell Biol 2004, 24:7402-7418. 